\title{
Development of maraviroc, a CCR5 antagonist for treatment of HIV, using a novel tropism assay
}

\author{
Elna van der Ryst, ${ }^{1}$ Jayvant Heera, ${ }^{2}$ James Demarest, ${ }^{3}$ and Charles Knirsch ${ }^{4}$ \\ ${ }^{1}$ The Research Network, Sandwich, United Kingdom. ${ }^{2}$ Pfizer Worldwide Research and Development, Groton, Connecticut. \\ ${ }^{3}$ ViiV Healthcare, Research Triangle Park, North Carolina. ${ }^{4}$ Pfizer Worldwide Research and Development, Pearl River, New \\ York
}

Address for correspondence: Charles Knirsch MD, MPH, 401 N. Middletown Road, Pearl River, NY 10965.

Charles.Knirsch@pfizer.com

\begin{abstract}
Assays to identify infectious organisms are critical for diagnosis and enabling the development of therapeutic agents. The demonstration that individuals with a 32-bp deletion within the CCR5 locus were resistant to human immunodeficiency virus (HIV) infection, while those heterozygous for the mutation progressed more slowly, led to the discovery of maraviroc (MVC), a CCR5 antagonist. As MVC is only active against CCR5-tropic strains of $\mathrm{HIV}$, it was critical to develop a diagnostic assay to identify appropriate patients. Trofile ${ }^{\mathrm{TM}}$, a novel phenotypic tropism assay, was used to identify patients with CCR5-tropic virus for the MVC development program. Results of these clinical studies demonstrated that the assay correctly identified patients likely to respond to MVC. Over time, the performance characteristics of the phenotypic assay were enhanced, necessitating retesting of study samples. Genotypic tropism tests that have the potential to allow for local use and more rapid turnaround times are also being developed.
\end{abstract}

Keywords: HIV; tropism; diagnostic; antiretroviral; assay

\section{Introduction and background}

Emergence of an infectious disease associated with a newly identified organism often leads to development of diagnostic tests together with a search for new therapeutics, and eventually to their discovery and development. Highly active antiretroviral therapy (HAART) for the treatment of infection with human immunodeficiency virus type 1 (HIV-1) became a reality with the introduction of protease inhibitors (PIs) used in combination with nucleoside reverse transcriptase inhibitors (NRTIs). ${ }^{1,2}$ The development of quantitative and highly sensitive HIV-1 plasma RNA assays, and the demonstration that HIV-1 RNA was a key prognostic factor for disease progression and death, ${ }^{3}$ greatly facilitated the development of further antiretroviral drugs, as plasma HIV-1 RNA was accepted as a surrogate marker for clinical end points (including opportunistic infections and death) that required larger sample sizes for clinical trials. ${ }^{4}$ Diagnostic assays to determine suitable HAART regimens for individual patients are widely used in HIV therapy. These include phenotypic and genotypic assays to evaluate virus susceptibility ${ }^{5}$ and genotypic tests to evaluate increased susceptibility to specific adverse reactions, such as HLA-B ${ }^{\star} 5701$ testing to predict hypersensitivity to abacavir. ${ }^{6}$

The introduction of HAART greatly reduced HIV-1-associated mortality and morbidity. However, toxicity of drugs, leading to poor compliance ${ }^{7}$ and development of resistance, ${ }^{8}$ results in treatment failure in a significant proportion of patients. This leads to a continued need for new drugs with new mechanisms of action. The HIV entry process is an attractive target for therapeutic intervention. The first step is binding of virus envelope gp120 to CD4 on the cell surface. This process leads to a conformational change in gp120 that allows binding to a chemokine receptor (CC chemokine receptor 5 (CCR5) or CXC chemokine receptor 4 (CXCR4)), which acts as a coreceptor. This is followed by fusion of the virus envelope with the host cell membrane (Fig. 1A). ${ }^{9}$ 
A
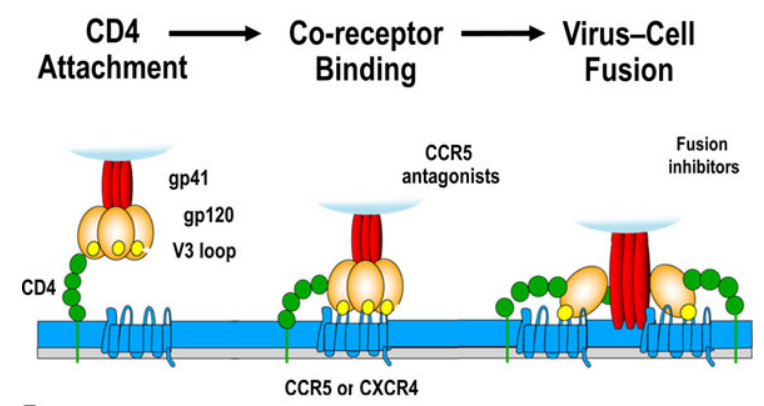

B

Pure $\mathrm{X} 4$

$$
\begin{aligned}
& { }_{x}^{x}{ }_{x}^{x}{ }_{x}^{x}{ }_{x}^{x} \\
& { }_{x}^{x}{ }_{x}^{x}{ }_{x}^{x} \\
& { }_{D}^{D} D_{D}^{D} D_{D}^{D} D_{D}^{D} \\
& \text { D D D D D } \\
& \begin{array}{llll}
R & R & R & R \\
R & R
\end{array}
\end{aligned}
$$

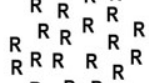

$$
\begin{aligned}
& R R R^{R}
\end{aligned}
$$

R5

\section{Mixed}

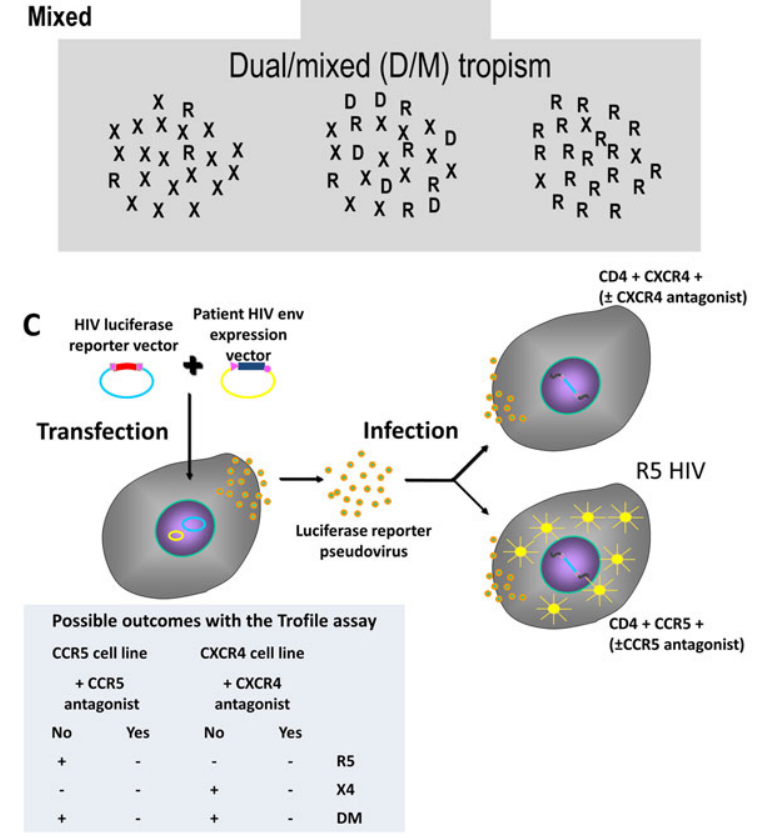

Figure 1. (A) HIV-1 cell entry process and potential molecular targets for antiretroviral compounds. Adapted from Ref. 9. (B) Possible tropism patterns that could be found in HIV-1-infected patients. R, CCR5 tropic (R5); X, CXCR4 tropic (X4); D, dual tropic. (C) Schematic diagram of the Trofile HIV-1 tropism assay. An HIV genomic luciferase reporter vector and a patient-specific ENV vector are cotransfected into HEK-293 cells to produce pseudovirus-expressing patient-derived envelope. Pseudovirus is added to U87 cells expressing either CCR5 or CXCR4. Infection is demonstrated by the addition of luciferase substrate and detection of luminescence, as is depicted here for $\mathrm{R} 5$ virus. X4 virus will result in infection of CXCR4 ${ }^{+}$cells only, while D/M virus populations will cause infection of both cell types. The infection is also performed in the presence of coreceptor antagonists to confirm specificity of the luminescence detected. The insert summarizes possible outcomes with the Trofile assay. Adapted from Ref. 15.

The virus population from an individual patient may contain only obligate CCR5-tropic (R5) or CXCR4-tropic (X4) strains, or dual-tropic strains
(R5X4) that can bind both CCR5 and CXCR4. A patient sample may also contain a heterogenous population of viruses with different tropism, 
termed mixed tropism (Fig. 1B). ${ }^{10}$ Dual- and mixedtropic virus populations are collectively termed dual/mixed (D/M). ${ }^{10}$ The discovery that individuals homozygous for the CCR $5 \Delta 32$ mutation are highly protected from infection with R5 HIV-1 strains ${ }^{11,12}$ and that heterozygotes have reduced disease progression ${ }^{13,14}$ led to a number of groups initiating research programs targeting the interaction between HIV and CCR5. However, in parallel, it was critical to develop a companion diagnostic assay to enable selection of patients most likely to benefit from these therapies: those with R5 virus.

Here, we describe the development of the CCR5 antagonist maraviroc (MVC) using a novel phenotypic assay to determine HIV-1 coreceptor tropism. The impact of improvements to the performance characteristics of the assay on the MVC development program is also discussed. The development of next-generation genotypic tropism tests that are convenient to use and can be closer to point of care would further simplify the use of MVC and facilitate integration of tropism testing into routine viral susceptibility testing.

\section{Trofile HIV-1 coreceptor tropism assay}

Trofile $^{\mathrm{TM}}$ is a phenotypic HIV-1 coreceptor tropism diagnostic assay developed by Monogram Biosciences (South San Francisco, CA) to facilitate development of coreceptor antagonists and to enable physicians to identify appropriate treatment regimens for patients presenting in their clinics.

Full-length HIV-1 envelope clones are created from patient plasma or serum using a reverse transcriptase polymerase chain reaction (RT-PCR). These clones are transferred into an expression vector (pCXAS-PXMX) to create patient envelope expression libraries that are transfected into HEK293 cells together with an envelope-deleted HIV-1 NL4-3 genomic vector (RTV1.F-lucP.CNDO $\Delta U 3$ ) expressing a luciferase reporter. The resulting luciferase reporter pseudovirus population expressing patient-derived envelope is then used to infect CD4 ${ }^{+}$U-87 cells expressing either CCR5 or CXCR4 receptors. Infection of target cells is evaluated by addition of a luciferase substrate and quantitation of the resulting luminescence. To demonstrate specificity, infection of target cells is also performed in the presence of CCR5 or CXCR4 antagonists (Fig. 1C). ${ }^{15,16}$ The assay determines whether the virus population found in a patient is $\mathrm{R} 5, \mathrm{X} 4$, or $\mathrm{D} / \mathrm{M}$ tropic (Figs. $1 \mathrm{~B}$ and $\mathrm{C}$ ).

\section{Assay characteristics and validation}

Monogram Biosciences' Clinical Reference Laboratory is accredited by the American College of Pathologists, and the assay was formally validated according to regulations specified by the Clinical Laboratory Improvement Amendments (CLIA). This included characterization of the accuracy, precision (intra-assay comparison), reproducibility (interassay comparisons), specificity, and sensitivity of the assay. Validation experiments demonstrated that the assay is sensitive and reproducible and able to accurately determine the coreceptor tropism of HIV-1 from patient plasma samples. The assay was able to amplify envelope from $>95 \%$ of samples that had HIV-1 RNA levels of $>1000$ copies $/ \mathrm{mL}$. Additionally, the assay can determine tropism to virus from all the HIV-1 subtypes. Mixing experiments indicated that the assay was able to detect minority X4 or R5 populations with $100 \%$ sensitivity when they constituted $10 \%$ of the population, while a population representing $5 \%$ of the total population could be detected with $85 \%$ sensitivity (Table 1$).{ }^{15}$

\section{Development of the enhanced-sensitivity Trofile assay}

Limited detection of minority variants is the common feature of all currently used population-based HIV-1 resistance assays, both phenotypic and genotypic, and the validation results from the Trofile assay are consistent with this. ${ }^{5,15}$ However, as treatment with CCR5 antagonists can lead to treatment failure due to emergence of a preexisting minority CXCR4-using virus population, efforts were made to improve the sensitivity of the assay for detection of minor variants. Several modifications, including manipulation of virus input levels, degree of coreceptor expression, and transfection and infection conditions, were evaluated. From these experiments, a combination of conditions designed to enhance $\mathrm{D} / \mathrm{M}$-tropic virus infection of $\mathrm{CXCR} 4^{+}$ cells was selected. No changes were made to the RTPCR and vector-construction steps. ${ }^{16}$ Validation experiments demonstrated that the performance characteristics of the enhanced sensitivity assay (ES Trofile) was similar to that of the original Trofile assay with regards to accuracy, precision, reproducibility, and viral load requirement (Table 1). However, experiments across 576 assays (288 with 
Table 1. Performance characteristics of the original and enhanced-sensitivity Trofile assays ${ }^{15,16}$

\begin{tabular}{|c|c|c|}
\hline & Original Trofile assay & Enhanced sensitivity Trofile assay \\
\hline Accuracy & $\begin{array}{l}100 \% \text { ( } 38 \text { test viruses from multiple } \\
\text { subtypes) }\end{array}$ & $\begin{array}{l}100 \% \text { ( } 16 \text { viruses from multiple subtypes } \\
\text { and } 30 \text { clonally analyzed patient samples) }\end{array}$ \\
\hline Precision & $100 \%$ & $100 \%$ \\
\hline Reproducibility & $100 \%$ & $99 \%$ \\
\hline Viral load requirement ${ }^{a}$ & 1000 copies $/ \mathrm{mL}$ & 1000 copies $/ \mathrm{mL}$ \\
\hline $\begin{array}{l}\text { Sensitivity for detection of minority } \\
\text { populations }\end{array}$ & $\mathrm{X} 4$ and $\mathrm{R} 5100 \%$ at $10 \%$ mixture & $\begin{array}{l}\mathrm{X} 4100 \% \text { at } 0.3 \% \text { mixture and R5 } 100 \% \text { at } \\
5 \% \text { mixture }\end{array}$ \\
\hline Specificity of amplification & $\begin{array}{l}\text { No false negative or false positive } \\
\text { amplifications observed }\end{array}$ & NA \\
\hline
\end{tabular}

Note: Adapted from Ref. 16. NA = not applicable.

${ }^{a_{95} \%}$ amplification success rate.

the ES Trofile assay and 288 with the original assay for comparison) to evaluate detection of low levels of minor variants demonstrated that the enhanced assay detected $\mathrm{X} 4$ env clones in $100 \%$ of assays when they constituted $0.3 \%$ of the population and R5 env clones in $100 \%$ of assays when present at $5 \%$ of the population, thereby confirming a significant improvement in sensitivity to detect X4 minority variants (Table 1). ${ }^{16}$ This assay has now replaced the original Trofile assay.

\section{Discovery and development of maraviroc}

Following the demonstration of the importance of the CCR5 receptor for HIV-1 entry, ${ }^{1-14}$ and based on the fact that $G$ protein-coupled receptors have historically been tractable to potent, selective, lowdose small-molecule drugs, Pfizer Global Research and Development (Sandwich, UK) initiated a CCR5 antagonist discovery program. ${ }^{17}$

High-throughput screening of the Pfizer compound file using a chemokine radioligand-binding assay resulted in the identification of an imidazopryridine lead molecule (UK-107543). An intensive medicinal chemistry program to optimize binding potency against the receptor, antiviral activity, absorption, and pharmacokinetics, and selectivity against human cellular targets resulted in the identification of MVC (formerly UK-427857) as a promising candidate for further development. Mechanism-of-action studies confirmed that MVC blocked the binding of virus gp120 to CCR5 and acted as a slow-offset functional antagonist of CCR5. MVC had no adverse effects in cell-based cytotoxicity studies, was highly selective for CCR5, and was predicted to have human pharmacokinetics consistent with once-daily (QD) or twice-daily (BID) dosing. It also demonstrated potent antiviral activity against all R5 HIV-1 strains tested. ${ }^{17}$

Preclinical safety studies of MVC revealed no significant safety concerns, and a phase I clinical program in healthy volunteers was initiated. ${ }^{18,19}$ Data from these studies indicated that MVC was safe and well tolerated in doses up to $900 \mathrm{mg}$ QD and $300 \mathrm{mg}$ $\mathrm{BID}$, did not influence the activity of major drugmetabolizing enzymes, and had an acceptable pharmacokinetic profile. ${ }^{19}$

\section{Phase Ila proof-of-concept studies}

On the basis of the favorable safety and pharmacokinetic profile of MVC demonstrated in the healthy volunteer studies, a phase IIa proof-of-concept program was initiated. ${ }^{20}$ However, as MVC was targeted for patients infected with R5 HIV-1, a diagnostic assay was needed that could reliably identify appropriate patients for inclusion in clinical studies. To this purpose, the newly developed Trofile (original) assay was identified as appropriate for clinical use.

A total of 82 patients prescreened for the absence of CXCR4-using virus, using the Trofile assay, were randomized to receive MVC monotherapy (doses from $25 \mathrm{mg}$ QD to $300 \mathrm{mg}$ BID) or placebo for 10 days with follow-up until day 40. MVC was well tolerated and mean viral load change from baseline to day 11 ranged from $-0.43 \log 10$ copies $/ \mathrm{mL}$ (25 mg QD) to $-1.60 \log 10$ copies/mL (300 mg BID). At doses of $100 \mathrm{mg}$ BID and above, all patients, with the exception of one patient with $\mathrm{D} / \mathrm{M}$ virus at baseline (who was accidentally enrolled owing to a sample-switching error at screening and had no 
response), experienced a maximal reduction in viral load of $>1.0 \log 10$ copies $/ \mathrm{ml}^{10,20}$

The Trofile assay was also used for sequential analysis of virus tropism for all patients at days 1, 11, and 40 (30 days posttreatment). Changes in virus tropism were seen in two patients receiving MVC. In one patient, there was transient emergence of $\mathrm{D} / \mathrm{M}$ virus on day 11 , but on day 40 only $\mathrm{R} 5$ virus could be detected. In the second patient, $\mathrm{D} / \mathrm{M}$ virus was detected on day 11 and remained detectable at follow-up until day 433, when HAART was initiated. ${ }^{10,20}$ Phylogenetic analysis of virus envelope clones from both patients demonstrated that the CXCR4-using virus emerged from a preexisting population rather than through a switch in coreceptor use of a R5 clone. ${ }^{10}$

Altogether, these results not only demonstrated the potential utility of MVC in the treatment of HIV, but also that the Trofile assay could be successfully used to identify patients likely to respond to a CCR5 antagonist and to evaluate changes in virus tropism during and after MVC treatment.

\section{Maraviroc phase IIb/III clinical development program}

Following the successful demonstration of proof of concept for MVC, a registrational phase IIb/III clinical development program evaluating MVC in doses of $300 \mathrm{mg}$ (or equivalent, depending on concomitant drugs) QD and BID was initiated. The program consisted of four major clinical studies (Table 2). Study A4001026 (MERIT) compared MVC and efavirenz, both with zidovudinelamivudine, in antiretroviral-naive patients with R5 HIV-1 infection. ${ }^{21}$ Studies A4001027 and A4001028 (MOTIVATE 1 and 2) evaluated MVC QD or BID plus optimized background therapy (OBT) versus placebo plus OBT in highly treatment-experienced patients with R5 virus at screening. ${ }^{22}$ A total of 4974 patients were screened for the MERIT and MOTIVATE studies using the Trofile assay. ${ }^{21,22}$

It was important to evaluate the safety of MVC in patients with CXCR4-using virus, as tropism assays may not detect minority CXCR4-using virus populations, and these patients may inadvertently be treated with a CCR5 antagonist. Therefore, patients who were excluded from entering the MOTIVATE studies because they had non-CCR5-using virus (CXCR4-using virus or nonphenotypable virus) at screening, and who met other inclusion criteria, were offered the opportunity to participate in a phase IIb safety study (A4001029). The study design was similar to that of the MOTIVATE studies (Table 2). ${ }^{23}$

Results of studies in treatment-experienced patients. Pooled 48-week data from the MOTIVATE studies demonstrated a significant virologic benefit for patients receiving OBT with MVC compared to those receiving OBT only (Fig. 2A). MVC treatment also provided a significant immunological benefit demonstrated by a significantly higher increase in mean $\mathrm{CD} 4^{+}$cell count (113 and 122 cells $/ \mathrm{mm}^{3}$ for MVC QD and BID, respectively, compared to 54 cells $/ \mathrm{mm}^{3}$ for OBT only), and had a safety profile similar to placebo. ${ }^{22}$

The 24-week results from study A4001029 demonstrated that MVC was safe and well tolerated in a population of patients with non-CCR5-tropic HIV-1 and resulted in a significantly higher increase in mean $\mathrm{CD}^{+}{ }^{+}$cell count from baseline $(60$ and 62 cells $/ \mathrm{mm}^{3}$ for MVC QD and BID, respectively, compared to 36 cells $/ \mathrm{mm}^{3}$ for OBT only). However, in contrast to what was seen in the MOTIVATE studies, no significant virologic benefit compared to placebo was seen for patients with non-CCR5-tropic virus who received MVC (Fig. 2B). ${ }^{23}$

The data from these three studies demonstrated that a patient population identified as having R5 HIV-1 by the Trofile assay is likely to experience significant virologic benefit from treatment with MVC, while those identified with non-CCR5-tropic HIV-1 did not experience significant benefit. ${ }^{22,23}$ The results from these studies led to MVC (300 mg BID) receiving approval for use (in combination with other antiretroviral drugs) in both the United States and Europe in the treatment of antiretroviralexperienced adults with R5 HIV-1 infection. ${ }^{24,25}$

Results in treatment-naive patients. The MERIT study compared QD and BID doses of MVC with efavirenz in patients with R5 HIV-1 infection. The MVC QD treatment arm was discontinued following an interim analysis of the first 205 patients at week 16, as it did not meet predefined noninferiority criteria to efavirenz. Data from the 48week analysis demonstrated a favorable safety profile and significantly higher $\mathrm{CD}^{+}{ }^{+} \mathrm{T}$ cell increases in patients receiving MVC. However, only $65 \%$ of MVC-treated patients, compared to $69 \%$ of efavirenz-treated patients, achieved an HIV-1 RNA 
Table 2. MVC phase IIb/III clinical development $\operatorname{program}^{21-23}$

\begin{tabular}{lllll}
\hline & \multicolumn{2}{c}{ R5 patients } & \\
\cline { 2 - 4 } & \multicolumn{1}{c}{ ARV naive } & ARV experienced & Non-R5 patients \\
\hline Study number/name & A4001026 MERIT & A4001027 MOTIVATE 1 & A4001028 MOTIVATE 2 & A4001029 \\
Phase & IIb $\rightarrow$ III & IIb/III & IIb/III & IIb \\
Design & MVC vs. EFV + CBV & OBT add-on & OBT add-on & OBT add-on \\
Randomization & $1: 1: 1$ & $2: 2: 1$ & $2: 2: 1$ & $1: 1: 1$ \\
Primary end point & $\%$ HIV-1 RNA $<400 /<50$ & $\Delta$ VL at week 24/48 & $\Delta$ VL at week 24/48 & $\Delta$ VL at week \\
& copies/mL at week 48/96 & & & $24 / 48$ \\
Randomized & 917 & 601 & 474 & 190 \\
\hline
\end{tabular}

ARV, antiretroviral; MVC, maraviroc; EFV, efavirenz; CBV, combivir (zidovudine plus lamivudine); HIV, human immunodeficiency virus; OBT, optimized background therapy; RNA, ribonucleic acid; VL, viral load.

of $<50$ copies $/ \mathrm{mL}$. This result did not meet the preset criterion for noninferiority at $10 \%$ threshold level (Fig. 2C). ${ }^{21}$

Since an enhanced-sensitivity Trofile (ES Trofile) assay had been developed in the interim, ${ }^{16}$ it was decided to evaluate whether use of a more sensitive assay to exclude patients with minority CXCR4using virus populations would have improved the outcome in the MVC treatment group. Reevaluation of the MERIT screening samples revealed that $107 / 721$ (14.8\%) patients would have been identified as having CXCR4-using virus if this assay had been used for the screening process. Subsequently, a post hoc reanalysis of the data was performed, excluding these patients. The post hoc reanalysis resulted in greater response rates in the MVC arm at week 48 , which now fell within the criteria defining non-inferiority (Fig. 2C). ${ }^{21}$ These data clearly demonstrate the improved clinical utility of the ES Trofile assay for selecting patients most likely to respond to treatment with a CCR5 antagonist. MVC is also approved for the treatment of antiretroviralnaive patients with R5 HIV-1 in the United States. $^{24}$

\section{Evaluation of reasons for virologic failure}

There are two potential pathways of virologic escape from CCR5 antagonists: selection of R5 virus that can use the drug-bound CCR5 receptor to enter host cells; and "un-masking" of preexisting CXCR4using virus populations through selective drug pressure by the CCR 5 antagonist. ${ }^{10,26}$ The Trofile assay was used to evaluate virus tropism during treatment in the MVC phase IIb/III studies at prespecified time points and at time of virologic failure, provided that HIV-1 RNA was present at $>500$ copies/mL. ${ }^{21,23,27}$
Of the 1042 patients in the MOTIVATE studies who had R5 tropism results at screening, 79 patients (8\%) had evidence of D/M virus at baseline, reflecting the limited sensitivity for minority viral species of the original Trofile assay. ${ }^{15}$ Of the 133 patients who failed MVC treatment and had R5 virus at baseline, 76 (57\%) had evidence of CXCR4-using virus at time of failure, compared to just 6/95 (6\%) who failed on OBT only (placebo). ${ }^{27}$ This is consistent with selective pressure from MVC and indicates that unmasking of preexisting CXCR4-using virus populations is the major mechanism of viral escape in this population.

Data from the post hoc reanalysis of the MERIT study using the ES Trofile assay indicated that of the 29 patients who received MVC and who had virologic failure, 9 (31\%) had evidence of CXCR4using virus at failure. ${ }^{21}$

\section{Further assay development}

While technical advances enhanced the performance of the original Trofile assay, logistical aspects continued to put constraints on the widespread use of this test for managing patient care. With the LabCorp acquisition of Monogram Biosciences in 2009, 2 years after the launch of MVC (Selzentry $\left.{ }^{\mathrm{TM}}\right)$, clinicians could use the LabCorp clinical/laboratory network to get samples more easily to the Monogram Biosciences laboratory, especially in the United States.

Outside the United States, shipment logistics still affected assay turnaround time, and this, together with cost, continued to limit the widespread clinical use of Trofile. This led to initiatives to develop other phenotypic assays based on recombinant 
A

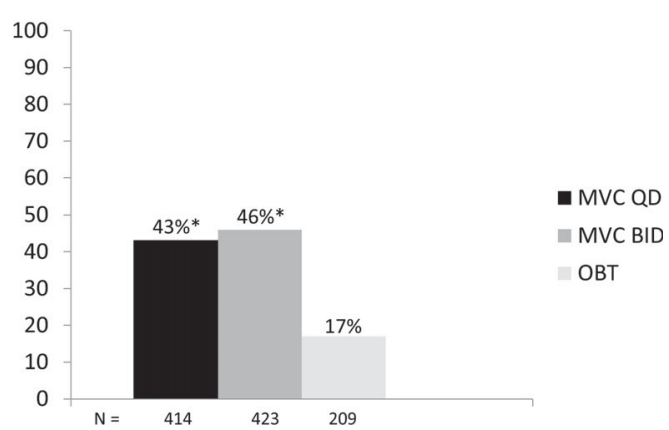

B

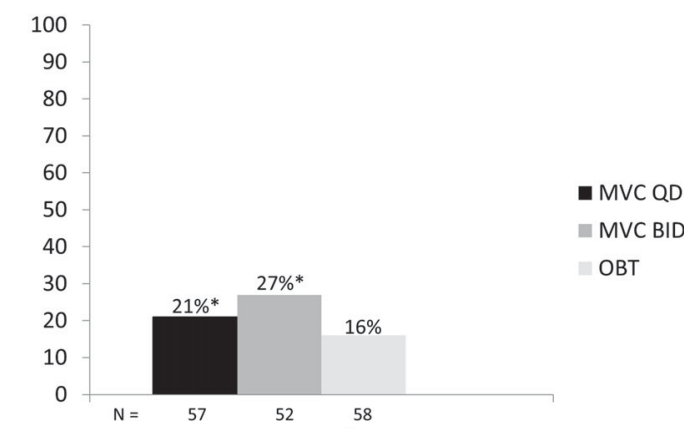

C

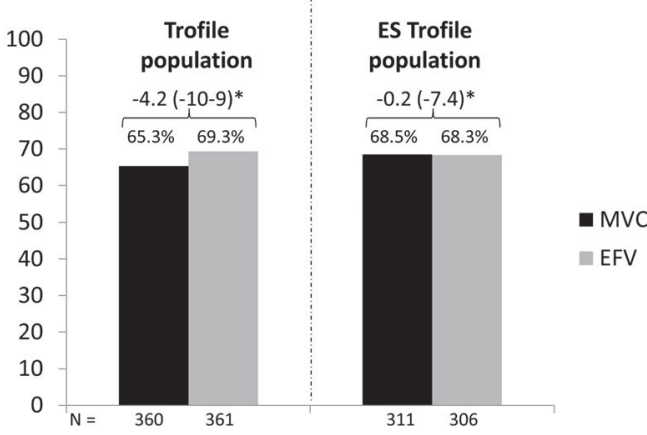

Figure 2. (A) MOTIVATE 1 and 2 combined analysis: proportions of patients achieving HIV-1 RNA $<50$ copies/mL at week 48 . HIV-1-infected patients with $\mathrm{R} 5$ virus and tripleclass experience (and/or triple-class resistance) were randomized to receive MVC QD or BID or placebo; all in combination with an OBT regimen, consisting of 3-6 antiretroviral drugs, selected by the investigator. MVC, maraviroc; QD, once daily; BID, twice daily. ${ }^{\star} P<0.001 .{ }^{22}$ (B) Study A4001029: proportions of patients achieving HIV-1 RNA $<5$ 0 copies/mL at week 24 . ${ }^{\star}$ Difference from placebo for MVC QD and MVC BID were 7 (95\% CI: -7 to 20$)$ and 11 (95\% CI: -3 to 26 ), respectively. ${ }^{23}$ (C) MERIT: proportions of patients achieving HIV-1 RNA $<50$ copies $/ \mathrm{mL}$ at week 48 in the primary analysis and the post hoc reanalysis using the ES Trofile assay. Treatment-naive HIV-1-infected patients with R5 virus were randomized to receive MVC twice daily or efavirenz once daily, both in combination with combivir. ${ }^{\star}$ Difference adjusted for randomization strata with lower bound of the onesided $97.5 \% \mathrm{CI}^{21}$ virus particles targeting a variety of envelope regions. Assays include Virco ${ }^{\circledR}$ Type HIV-1 (Janssen Diagnostics, Belgium), ${ }^{28}$ Phenoscript ${ }^{\circledR}$ (Eurofins, Belgium/Specialty Laboratories, NJ), ${ }^{29}$ PhenX-R (InPheno AG, Switzerland), ${ }^{30}$ the Toulouse Tropism Test, ${ }^{31}$ and the TROCAI test. ${ }^{32}$

\section{Genotypic tropism assays}

However, all phenotypic assays are relatively expensive and time-consuming and require samples to be shipped to specialized laboratories. Genotypic tropism assays using the HIV-1 V3 loop sequence to predict tropism run by local/regional laboratories were therefore developed in Europe and other regions as a more practical alternative for patient care. Owing to the inherent genetic variability of the HIV envelope V3 loop and the lack of true gold standards for the detection of R5-, DM-, and X4tropic variants with sufficient diversity to cover that seen in patients, both improvements in sequencing technology (to amplify the V3 loop and efficiently read the sequence in the diverse region) and algorithm development (to be able to predict tropism from the derived sequence) were required.

While many groups worked together formally and informally to advance the knowledge and technical capabilities required for a reliable approach to a genotypic tropism assay, in the interest of brevity, we highlight here a few illustrative examples. These approaches utilized a retrospective analysis of samples from MERIT and/or MOTIVATE/A4001029, and include both population-based Sanger sequencing as well as next-generation ultradeep sequencing (UDS).

\section{Population-based Sanger sequencing}

Samples from subjects enrolled in MVC clinical trials were retrospectively tested using populationbased Sanger sequencing to determine concordance and predictive values for clinical outcome. The MERIT and A4001078 studies in treatment-naive patients and the MOTIVATE and A4001029 studies in treatment-experienced patients were useful in this regard.

In MERIT, $84 \%(292 / 351)$ of subjects who received MVC were concordant as R5/R5 or nonR5/non-R5 by both ESTA and genotype; 283 (81\%) R5/R5 and 9 (3\%) non-R5/non-R5. In terms of virologic outcome, the percentages of subjects achieving HIV-1 RNA levels $<50$ copies/mL were consistent with the assay concordance; approximately $60 \%$ 
over 96 weeks for R5/R5 and approximately $20 \%$ or less for non-R5/non-R5. ${ }^{33}$ Interestingly, for those subjects who were discordant (R5 genotype/non-R5 ESTA, $n=39$ and R5 ESTA/non-R5 genotype, $n=$ 20 ), the response rate for either group was lower but similar to the R5/R5 patient group, suggesting that for these subjects neither assay was fully accurate in predicting $\mathrm{R} 5$ tropism. ${ }^{33}$

These data in the discordant groups highlight a few of the challenges for genotypic and phenotypic tropism assays. First, there is no true gold standard, as demonstrated by the fact that the response rate of patients with discordant results was not driven by either assay: the response curves overlapped and were not separated on the basis of being R 5 by one assay over the other. Second, as was seen with the advances from the original Trofile to the current ES Trofile assay, the threshold for detection of minority species may be relevant for tropism prediction. In general terms, Sanger sequencing methods have a $15 \%-20 \%$ threshold for detection of a minority species. Optimization of both the laboratory method and the algorithm settings for tropism prediction has improved the sensitivity of a given genotypic method. For the latter, the retrospective analysis of study A4001078 that used the ES Trofile assay for tropism screening illustrates this point. ${ }^{34}$ Screening samples from all 199 subjects evaluated for possible enrolment in A4001078 were retrospectively evaluated by a Sanger sequencing method using different false positive rate (FPR) thresholds with the geno2pheno algorithm. A higher FPR increases sensitivity for $\mathrm{X} 4$ variants, but reduces specificity. In this study, the percentage of subjects classified as X4 increased with increasing FPR values (5.75\% FPR: 15.1\% through 10\% FPR: $23.1 \%$ and 20\% FPR: $37.7 \%)$. For the 144 samples that were R5 by ESTA, $132(91.7 \%)$ and $120(83.3 \%)$ were R5 by genotype with FPRs of $5.75 \%$ and $10 \%$, respectively. ${ }^{34}$ Similar results were obtained for other studies. ${ }^{35,36}$

However, an association of tropism determination by the given assay with MVC response is ultimately a better measure of assay performance than concordance with another assay, as it is a direct measure of clinical utility. Therefore, a positive predictive value (PPV), defined as the likelihood of a patient having a positive virologic response with MVC when virus tropism is defined as R5, may provide a better way of comparing assays. A recent study evaluating a Sanger sequencing genotyping method with FPR $10 \%$ demonstrated a concordance of $72 \%$, with model-based estimates of PPV of $79.1 \%$ for genotype versus $76.3 \%$ for ES Trofile. ${ }^{37}$

\section{Next-generation ultradeep sequencing}

The threshold for detection of minor variants using UDS platforms is typically $<1 \%$. Though current availability and use of these platforms are generally for research purposes only, UDS may provide an alternative genotypic tropism methodology going forward.

The analysis of samples from MOTIVATE and A4001029 provide the opportunity to explore the sensitivity for detection and the clinical relevance of minority CXCR4-using variants. An initial blinded analysis was performed using the Roche 454 UDS platform. ${ }^{38}$ At the time of this analysis, only original Trofile data were available on the MOTIVATE and A4001029 samples; however, the analysis by virologic outcomes based on original Trofile and 454 UDS were similar. ${ }^{38}$

Subsequent to this analysis, Kagan et al. at Quest Diagnostics (Madison, NJ) performed an analysis with 327 subjects enrolled in either MOTIVATE or A4001029; ES Trofile results were available at this time. ${ }^{39}$ In this blinded analysis, Sanger sequencing was performed either in isolation or as a first step in a reflex assay to 454 next-generation sequencing; for the reflex approach, if a sample was called R5 by Sanger, the 454 analysis was performed, whereas if the Sanger result was non-R5, no further testing was conducted. The 454 UDS method, either alone or as part of the reflex format, had similar PPVs to ES Trofile (66\% for ES Trofile versus 65\% for either 454 UDS alone or in the reflex assay). ${ }^{39}$

\section{Short-term treatment with MVC}

In an alternative approach, Genebat et al. evaluated the correlation of virologic response to short-term (8 days) treatment with MVC to clinical outcome and the ES Trofile assay result. The data demonstrated a concordance of $93.5 \%$ between the ES Trofile assay and response to short-term MVC therapy. Additionally, all patients who subsequently went on to receive MVC as part of an optimized regimen had a good virologic response. ${ }^{40}$ However, the same group subsequently demonstrated a $15.2 \%$ discordance rate between ES Trofile and short-term MVC therapy in a larger group of patients with HIV-1 RNA $\geq 1000$ copies/mL. Four of the seven 
patients with discordant results were identified as having $\mathrm{D} / \mathrm{M}$ virus by ES Trofile. ${ }^{41}$ Consistent with these data, a recent study demonstrated that the response to short-term MVC treatment was similar in $\mathrm{R} 5$ and $\mathrm{D} / \mathrm{M}$ patients, casting doubt on the clinical validity of this approach. ${ }^{42}$

\section{Treatment guidelines for the use of tropism testing}

The United States has been slow to adopt genotypic tropism methods to guide MVC use. ${ }^{43}$ However, for the reasons outlined above, the EU guidelines on tropism testing have adopted genotypic tropism testing as a viable approach to managing the appropriate use of MVC as a part of HAART. ${ }^{44}$ This was driven by both the logistical concerns and the growing data sets using genotypic tropism tests. The retrospective analyses and the commercial availability of the reflex genotypic tropism assay have further contributed to this evolution. ${ }^{31}$ The use of tropism testing in other regions to guide appropriate use of MVC generally follows the EU and/or U.S. guidelines.

\section{Summary}

The development and utilization of a diagnostic assay to accurately determine HIV-1 coreceptor tropism was critical to the development of the CCR5 antagonist MVC. On the other hand, the results of the MVC registrational clinical studies demonstrated the utility of the Trofile assay for the selection of patients most likely to benefit from treatment with a CCR5 antagonist, thereby confirming both its clinical positive and negative predictive value. Retrospective evaluation of stored clinical samples from the MVC clinical studies have also been useful in the evaluation of genotypic tropism tests that are cheaper, more convenient to use, and can be utilized closer to the point of clinical care, allowing quicker turnaround times and more rapid clinical decision making. Altogether, scientific advances have led to more sensitive and accessible options for tropism testing, either as phenotypic- or genotypic-based methods, and this provides a good example of the simultaneous development of a new treatment for HIV and an enabling diagnostic assay.

\section{Acknowledgments}

The authors would like to thank all patients and investigators who participated in the MVC studies, as well as numerous colleagues at Pfizer and ViiV who contributed to the program. We also thank colleagues at Monogram Biosciences.

\section{Conflict of interest}

Charles Knirsch and Jayvant Heera are the employees of Pfizer Inc., and are partly compensated through stock/stock options. James Demarest is an employee of ViiV Healthcare. Elna van der Ryst was an employee of Pfizer Inc. at the time the MVC studies were conducted and currently provides consulting services to Pfizer.

\section{References}

1. Autran B., G. Carcelain, T.S. Li, et al. 1997. Positive effects of combined antiretroviral therapy on $\mathrm{CD}^{+} \mathrm{T}$ cell homeostasis and function in advanced HIV disease. Science 277: 112-116.

2. Collier A.C., R.W. Coombs, D.A. Schoenfeld, et al. 1996. Treatment of human immunodeficiency virus with saquinavir, zidovudine and zalcitabine. N. Engl. J. Med. 334: 1011-1017.

3. Mellors J.W., A. Munoz, J.V. Giorgi, et al. 1997. Plasma viral load and $\mathrm{CD}^{+}$lymphocytes as prognostic markers of HIV1 infection. Ann. Intern. Med. 126: 947-954.

4. U.S. Department of Health and Human Services, Food and Drug Administration, Center for Drug Evaluation and Research (CDER). 2002. Guidance for Industry. Antiretroviral drugs using plasma HIV RNA measurements_clinical considerations for accelerated and traditional approval.

5. Hirsch M., F. Brun-Vezinet, R.T. D’Acquila, et al. 2000. Antiretroviral drug resistance testing in adult HIV-1 infection. Recommendation of an International AIDS SocietyUSA panel. J. Am. Med. Acad. 283: 2417-2426.

6. Hetherington S., A.R. Hughes, M. Mosteller, et al. 2002. Genetic variations in HLA-B region and hypersensitivity reactions to abacavir. Lancet 359: 1121-1122.

7. d'Arminio M.A., A.C. Lepri, G. Rezza, et al. 2000. Insights into the reasons for discontinuation of the first highly active antiretroviral therapy (HAART) regimen in a cohort of antiretroviral naïve patients. ICONA study group, Italian cohort of anti-retroviral naive patients. AIDS 14: 499-507.

8. Richman D.D., S.C. Morton, T. Wrin, et al. 2004. The prevalence of antiretroviral drug resistance in the United States. AIDS 18: 1393-1401.

9. Moore J.P. \& R.W. Doms. 2003. The entry of entry inhibitors: a fusion of science and medicine. Proc. Nat. Acad. Sci. U.S.A. 100: 10598-10602.

10. Westby M., M. Lewis, J. Whitcomb, et al. 2006. Emergence of CXCR4-using human immunodeficiency virus type 1 (HIV1) variants in a minority of HIV-1-infected patients following treatment with the CCR5 antagonist maraviroc is from a pretreatment CXCR4-using reservoir. J. Virol. 80: 49094920.

11. Liu R., W.A. Paxton, S. Choe, et al. 1996. Homozygous defect in HIV-1 coreceptor accounts for resistance of some multiply exposed individuals to HIV-1 infection. Cell 86: 367-377. 
12. Samson M., F. Libert, B. Doranz, et al. 1996. Resistance to HIV-1 infection in caucasian individuals bearing mutant alleles of the CCR5 chemokine receptor gene. Nature 382: 722-725.

13. Dean M., M. Carrington, C. Winkler, et al. 1996. Genetic restriction of HIV-1 infection and progression to AIDS by a deletion allele of the CKR5 structural gene. Hemophilia growth and development study, multicenter AIDS cohort study, multicenter hemophilia cohort study, San Francisco city cohort, ALIVE. Science 274: 1856-1862.

14. Pasi K.J., C.A. Sabin, P.V. Jenkins, et al. 2000. The effects of the 32bp CCR-5 deletion on HIV transmission and HIV disease progression in individuals with heamophilia. $\mathrm{Br}$. J. Hematol. 111: 136-142.

15. Whitcomb J.M., W. Huang, S. Fransen, et al. 2007. Development of a novel single-cycle recombinant-virus assay to determine human immunodeficiency virus type 1 coreceptor tropism. Antimicrob. Agents Chemother. 51: 566-575.

16. Reeves J.D., E. Coakley, C.J. Petropoulos, et al. 2009. An enhanced sensitivity Trofile HIV coreceptor tropism assay for selecting patients for therapy with entry inhibitors targeting CCR5: a review of analytical and clinical studies. J. Viral Entry 3: 94-102.

17. Dorr P., M. Westby, S. Dobbs, et al. 2005. Maraviroc (UK427857), a potent, orally bioavailable, and selective small molecule inhibitor of chemokine receptor CCR 5 with broadspectrum anti-human immunodeficiency virus type 1 activity. Antimicrob. Agents Chemother. 49: 4721-4732.

18. Dorr P., B. Stammen \& E. van der Ryst. 2012. Discovery and development of maraviroc, a CCR5 antagonist for the treatment of HIV infection. In Case studies in Modern Drug Discovery and Development. R.G. Aslanian \& X. Huang, Eds.: 196-226. New York: Wiley.

19. Abel S., E. van der Ryst, M.C. Rosario, et al. 2008. Assessment of the pharmacokinetics, safety and toleration of maraviroc, a novel CCR5 antagonist, in healthy volunteers. Br. J. Clin. Pharmacol. 65(Supplement 1): 5-18.

20. Fätkenheuer G., A.L. Pozniak, M.A. Johnson, et al. 2005. Efficacy of short-term monotherapy with maraviroc, a new CCR5 antagonist in HIV-1 infected patients. Nat. Med. 11: 1170-1172.

21. Cooper D.A., J. Heera, J. Goodrich, et al. 2010. Maraviroc versus Efavirenz, both in Combination with Zidovudine/Lamivudine, for the Treatment of Antiretroviral-Naïve Subjects with CCR5-tropic HIV-1. J. Infect. Dis. 201: 803813.

22. Gulick R.M., J. Lalezari, J. Goodrich, et al. 2008. Maraviroc for previously treated patients with R5 HIV-1 Infection. N. Engl. J. Med. 359: 1429-1441.

23. Saag M., J. Goodrich, G. Fatkenheuer, et al. 2009. A doubleblind placebo-controlled trial of Maraviroc in treatmentexperienced patients infected with non-CCR5 tropic HIV-1: 24-week results. J. Infect. Dis. 11: 1638-1647.

24. ViiV Healthcare. 2014. Prescribing information and medication guide for Selzentry. http://www.viivhealthcare. com/media/70429/us_selzentry.pdf. March 1, 2014 Accessed November 5, 2014.

25. European Medicines Agency. 2007. Celsentri Summary of Product Characteristics. http://www.ema.europa.eu/docs/ en_GB/document_library/EPAR_-_Product_Information/
human/000811/WC500022190.pdf. September 18, 2007. Accessed November 5, 2014.

26. Westby M., C. Smith-Burchnell, J. Mori, et al. 2007. Reduced maximal inhibition in phenotypic susceptibility assay indicates that viral strains resistant to the CCR5 antagonist maraviroc utilize inhibitor-bound receptor for entry. J. Virol. 81: 2359-2371.

27. Fätkenheuer G., M. Nelson, A. Lazzarin, et al. 2008. Subgroup analysis of maraviroc in previously treated R5 HIV-1 infection. N. Engl. J. Med. 359: 1442-1445.

28. Van Baelen K., I. Vandenbroucke, E. Rondelez, et al. 2007. HIV-1 coreceptor usage determination in clinical isolates using clonal and population-based genotypic and phenotypic assays. J. Virol. Methods 146: 61-73.

29. Trouplin V., F. Salvatori, F. Capello, et al. 2001. Determination of coreceptor usage of human immunodeficiency virus type 1 from patient plasma samples using by using a recombinant phenotypic assay. J. Virol. 75: 251-259.

30. Hamy F., V. Vidal, S. Hubert, et al. Hybridization-based assay and replicative phenotyping as diagnostic platform for determination of co-receptor tropism. 5th European HIV Drug Resistance Workshop. March 28-30, 2007. Cascais, Portugal. Abstract 60.

31. Raymond S., P. Delobel, M. Mavigner, et al. 2010. Development and performance of a new recombinant virus phenotypic entry assay to determine HIV-1 coreceptor usage. J. Clin. Virol. 47: 126-130.

32. Gonzalez-Serna A., M. Leal, M. Genebat, et al. 2010. TROCAI (tropism coreceptor assay information): a new phenotypic tropism test and its correlation with Trofile enhanced sensitivity and genotypic approaches. J. Clin. Microbiol. 48: 4453-4458.

33. McGovern R.A., A. Thielen, S. Portsmouth, et al. 2012. Population-based sequencing of the V3-loop can predict the virological response to maraviroc in treatment-naive patients of the MERIT trial. J. Acquir. Immune Defic. Syndr. 61: 279-286.

34. Portsmouth S., S.R. Valluri, M. Däumer, et al. 2013. Correlation between genotypic (V3 population sequencing) and phenotypic (Trofile ES) methods of characterizing coreceptor usage of HIV-1 from 200 treatment-naïve HIV patients screened for study A4001078. Antiviral Res. 97: 6065.

35. Svicher V., R. D’Arrigo, C. Alteri, et al. 2010. Performance of genotypic tropism testing in clinical practice using the enhanced sensitivity version of Trofile as reference assay: results from the OSCAR Study Group. New Microbiol. 33: 195-206.

36. Recordon-Pinson P., C. Soulié, P. Flandre, et al. 2010. Evaluation of the genotypic prediction of HIV-1 coreceptor use versus a phenotypic assay and correlation with the virological response to maraviroc: the ANRS GenoTropism study. Antimicrob. Agents Chemother. 54: 3335-3340.

37. Heera J., S. Valluri, C. Craig, et al. 2014. First prospective comparison of genotypic vs phenotypic tropism assays in predicting virologic responses to Maraviroc (MVC) in a Phase 3 study: MODERN. J. Int. AIDS Soc. 17(S3): 19519.

38. Swenson L.C., T. Mo, W.W. Dong, et al. 2011. Deep sequencing to infer HIV-1 co-receptor usage: application to 
three clinical trials of maraviroc in treatment-experienced patients. J. Infect. Dis. 203: 237-245.

39. Kagan R.M., E.P. Johnson, M. Siaw, et al. 2012. A genotypic test for HIV-1 tropism combining Sanger sequencing with ultradeep sequencing predicts virologic response in treatment-experienced patients. PLoS One 7: e46334.

40. Genebat M., E. Ruiz-Mateos, J.A. León, et al. 2009. Correlation between the Trofile test and virological response to a short-term maraviroc exposure in HIV-infected patients. $J$ Antimicrob. Chemother. 64: 845-849.

41. Genebat M., E. Ruiz-Mateos, A. González-Serna, et al. 2011. Discordance rates between Trofile test and short-term virological response to maraviroc. Antiviral. Res. 89: 182-185.
42. Hernández-Novoa B., N. Madrid-Elena, F. Dronda, et al. 2014. Virological response to short-course maraviroc monotherapy does not predict viral tropism in HIV-1infected treatment-naive patients. J. Antimicrob. Chemother. 69: 1916-1919.

43. Department of Health and Human Services. 2014. Guidelines for the use of antiretroviral agents in HIV-1infected adults and adolescents. http://aidsinfo.nih.gov/ contentfiles/lvguidelines/adultandadolescentgl.pdf. November 13, 2014. Accessed November 15, 2014.

44. Vandekerckhove L.P., A.M. Wensing, R. Kaiser, et al. 2011. European guidelines on the clinical management of HIV-1 tropism testing. Lancet Infect Dis. 11: 394-407. 